\title{
Analysis of dynamic thermal processes in cement kilns by using the 2D and 3D numerical models
}

\author{
by V. G. Torgunakov, M. N. Sukhanov, V. P. Vavilov \\ Tomsk Polytechnic University, Russia, 634028, Tomsk, 28, Savinykh St., 3, \\ Tel.+7(3822)418712 Fax+7(3822)417281 E-mail:vavilov@introscopy.tpu.ru
}

\begin{abstract}
The 3D heat conduction numerical model is proposed for analyzing the opeartion of rotary kilns used in the production of cement. The usefulness of this model in solving an inverse diagnostic problem and compiling a map of significant defects is demonstrated.
\end{abstract}

\section{Introduction}

Thermal process modeling is widely used in the development of rotary kilns used in cement production $[1,2]$. In most models, at least in Russia, only a 1D or even one-point geometry is being used. However, simulating multi-dimensional thermal events becomes increasingly important due to growing use of IR thermography in monitoring kiln surface and evaluating kiln technical conditions [3].

We believe that a proper mathematical model of kiln inspection, along with the corresponding software, is indispensable in modern technology. Our earlier computer program was able to simulate a temperature distribution on both internal and external kiln surface, in particular, in the clinker area that is the most dangerous from a 'thermal' point of view [4]. This model included phenomena of heat conduction through a layered kiln shell and production of clinker (figure1).

The following factors were taken into account:

- convection and radiation on external surface,

- heat conduction within kiln shell;

- convection and radiation in the interaction between the internal kiln surface and the gas that flows inside a kiln,

- kiln rotation,

- mechanical motion of a raw product.

\section{Results and discussion}

The object (kiln) was represented as a set of Dirikhle cells constructed in the cylindrical 3D coordinates ( $r-\varphi-z$ geometry) [5]. The heat energy equations written for all cells formed the model of thermal processes within a kiln. Mathematically, the problem was reduced to solving a system of linear equations [6]. A more detailed description of the algorithm is given in [4].

The heat exchange between the kiln shell and flowing gas is modeled by using the known criteria of similarity (Nusselt's and Reynolds' criteria). Clinker motion speed and viscosity are determined by kiln inclination, as well as by kiln rotation speed [2]. These assumptions allowed simplifying the 3D model in order to obtain numerical results with longer time steps.

The analysis of thermal fluxes in both radial and axial directions has shown that, in a nondefect area, as well as in defect areas of large size, radial thermal fluxes dominate over lateral heat fluxes. Hence, a simplified 2D $(r-z)$ model can be introduced for kilns without 
defects or having defects of large size. In such model, only radial conductance was taken into account. The equivalent electrical circuit for the $k$-th cross-section is shown in figure 2.

Here: $U_{0}$ is the ambient temperature, $U_{1}$ - the temperature of the kiln external surface, $U_{2}$ and $U_{3}$ are the temperatures on the boundaries between kiln layers, $U_{4}$ is the temperature of the external surface, $U_{5}$ is the clinker temperature, $U_{6}$ is the gas temperature, $T_{0}$ is the clinker temperature in the $k+1$-th cross-section, $\mathrm{C}_{0}-\mathrm{C}_{7}$ are the thermal resistances between respective points.

This simplified model has also allowed solving the corresponding inverse problem, i.e. determining internal temperature by introducing in the solution values of external temperature that are experimentally measured by an IR thermographic device. The solution was first performed for the point with a maximum value of $k$ and then, consequently, for the next points with decreasing values of $k$. In each point, the temperatures $U_{0}$ and $U_{6}$, as well as all thermal resistances except $\mathrm{C}_{3}$, were considered as known. Both resistance $\mathrm{C}_{3}$ and internal kiln radius were found as a result of computations.

In figure 3, there is the internal surface profile crossing the area with a defect that extends from Point \#32 up to Point \#56. The defect extension is $60^{\circ}$. The selected profile crosses the defect in the middle. The results in figure 4 show the temperature distributions by the z-axis that correspond to the profile above and have been calculated by using both $3 \mathrm{D}$ and $2 \mathrm{D}$ models.

It is well seen from figure 4 that, in the non-defect area, temperature values are practically identical (data scatter not higher than $2^{\circ} \mathrm{C}$ ). However, data scatter in the defect area is considerable being explained with the fact that there is no heat conduction along both $z-$ and $\varphi$-coordinate within the kiln case.

Figure 5 presents the results of temperature visualization for the external surface obtained for the same input data by using both models. The image on the left shows the 3D distribution, the right image corresponds to the 2D model. The discrepancy between the two images is significant along defect boundaries.

The correctness of calculations was also verified by introducing 3D data into the inverse solution as input data.

In figure 6, there are two profiles presented: the original profile of the internal surface (solid line) and the profile calculated as a result of solving the inverse problem. A good profile coincidence takes place in both non-defect area and defect center, meanwhile there is a specific 'smashing' phenomenon occurring along defect boundaries.

The analysis has shown that temperature strongly depends on the following parameters: heat exchange coefficient on the external surface, ambient temperature and meteorology. The influence of external heat exchange is illustrated in figure 7.

Since a typical value of a heat exchange coefficient is in the range $15-40 \mathrm{~W} /\left(\mathrm{m}^{2} \cdot K\right)$, its influence on sound temperature is not too significant, meanwhile an error in determining a defect temperature could reach $100^{\circ} \mathrm{C}$ that leads to an error in determining the internal radius about $10 \mathrm{~cm}$. Hence, it is necessary to determine the heat exchange iteratively in the course of computations.

The influence of defect size on external temperature was analyzed within the 3D model by altering defect dimensions. A spatial step between two nodes was $0.125 \mathrm{~m}$, defect depth $0.06 \mathrm{~m}$. As it was expected, the smaller is the defect the lower is the temperature signal (see figure 8 ) that is the result of 3D heat diffusion around the defect. This effect will lead to further errors when determining a depth of small size defects.

A non-linear increase in external temperature with growing defect depth is illustrated with figure 9 (thermal insulation thickness was always $15 \mathrm{~cm}$ in numerical calculations).

The purpose of our theoretical analysis was calculating kiln external temperature. Of course, the same model can be used to study relationships between other parameters.

For a more practical use, we have developed the "Thermolnspector-1" and "Thermolnspector-2" computer programs intended for processing experimental data supplied 
by our "Introcon-04" line scanner. The latter system was developed at Tomsk Polytechnic University to be exclusively used in the inspection of rotary kilns (figure 10).

Figure 11 shows the example of data presentation provided by an "Introcon-04" system. Results of computer modeling are used to compile a defect map that exhibits defect with thermal insulation loss more than allowed.

\section{Conclusions}

- The proposed numerical model proved its validity in calculating both internal and external kiln temperature, as well in evaluating kiln internal radius.

- While identifying small-size defect, a high thermal conductance of kiln metallic shell is to be taken into account.

- The external surface thermal parameters are to be determined with a very high accuracy to ensure reasonable estimates of a kiln internal radius.

- The proposed model can be recommended not only for NDT purposes. It can be helpful in understanding the processes that occur in rotary kilns, as well as in designing new types of kilns. Numerical analysis is cheap and allows simulating any kiln regime, including that leading to possible accidents, meanwhile on-site experiments are typically cumbersome and long-continued.

\section{References}

[1] OBODOV (A.M.) and FIRSOVA (N.E.) - Passive thermal inspection of materials during their exploitation. Sov.J.NDT (Defectosopiya), 1991, No.11, pp.77-83.

[2] VOROBYV (Kh.S.) and MAZUROV (D.Ya.) - Thermal analysis of kilns and other equipment used in the production of cement. Vyshsya shkola Publish., , Moscow, 1962.-350 p. (in Russian).

[3] TORGUNAKOV (V.G.) - Two-stage system for inspecting industrial installations. In: Proc. VII Intern. Conf. on Optical, Microwave and Thermal NDT, Cherepovets, Russia, 1997, pp.46-47.

[4] SUKHANOV (M.S.) and TORGUNAKOV (V.G.) - Computer Model of Thermal Processes in a Cement Kiln. In: Proc. of the $7^{\text {th }}$ European Conference on Non-Destructive Testing. Denmark, 1998, pp.167-171.

[5] SOLOVYEV (A.V.) and SOLOVYEV (E.V.) - Solving gas-dynamical equations in cylindrical coordinates by using Dirikhle cells. Preprint of Institute of Applied Mechanics No.80, USSR Academy of Science, Moscow, 1986.-32 p. (in Russian).

[6] GODUNOV (S.K.) and SAMARSKY (V.S.) - Finite-difference schemes: introduction to the theory, Nauka Publish., Moscow, 1973.-400 p. (in Russian).

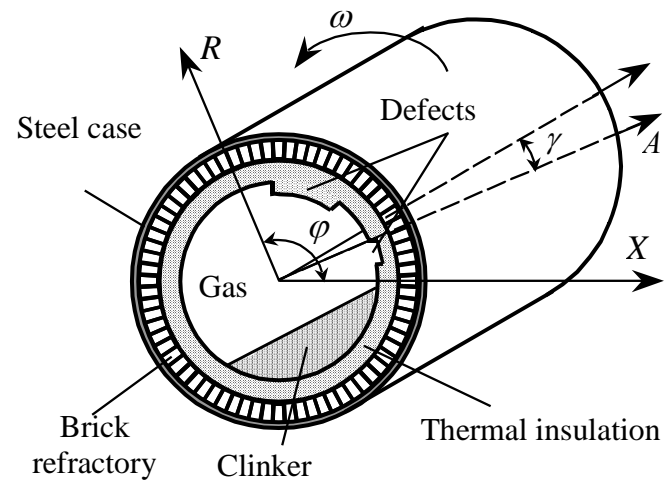

Fig.1. Rotary kiln for cement production 


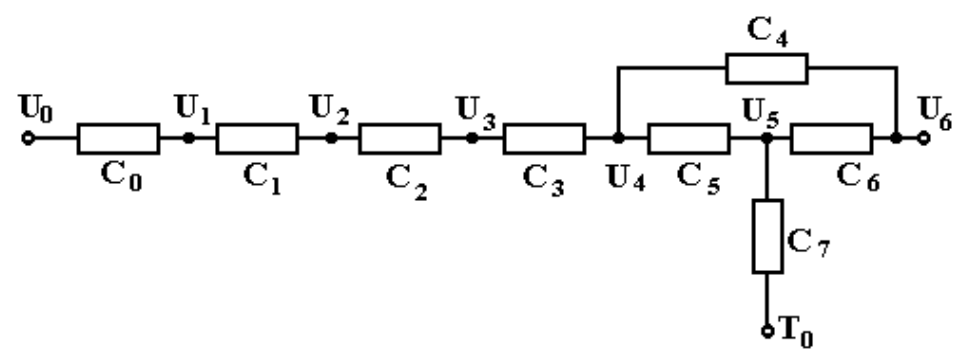

Fig.2. Equivalent electrical circuit for thermal processes
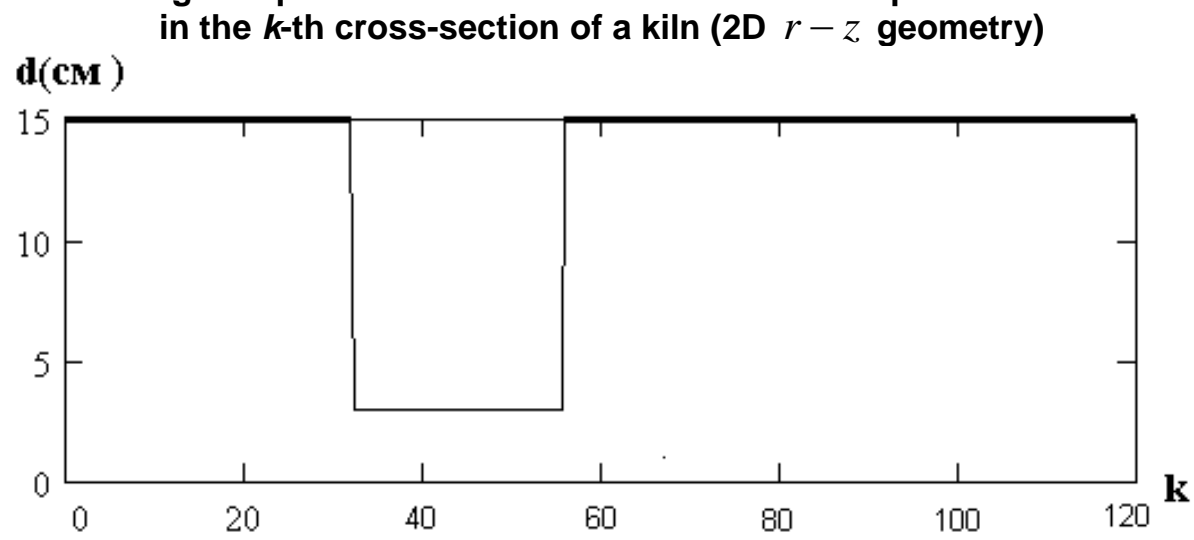

Fig.3. Profile of thermal insulation along the z-axis ( $k$ is the number of the calculated point)

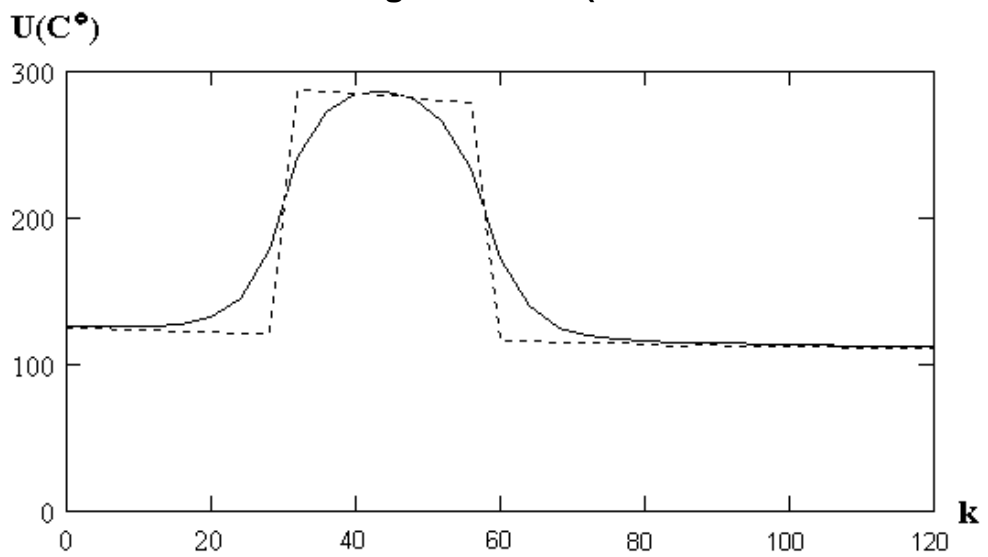

Fig.4. Comparison between temperature distributions calculated with the 3D (solid line) and 2D (dashed line) model

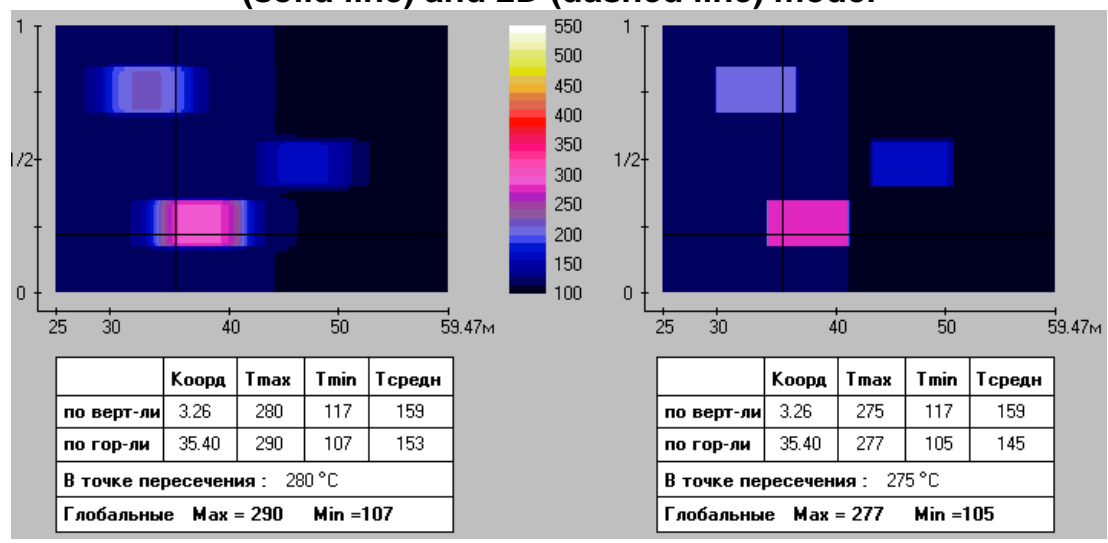

Fig.5. Visualization of the temperature distribution on the kiln external surface performed by using the 3D (on the left) and 2D (on the right) model 


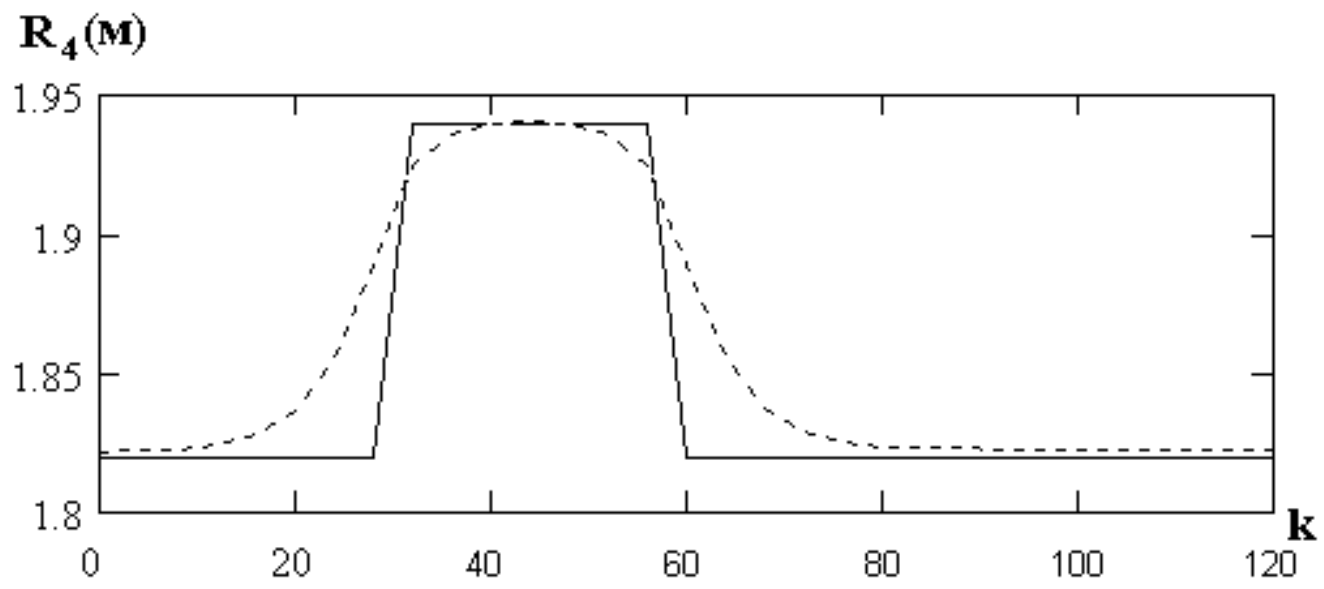

Fig.6. Comparison between the original profile (solid line) and the profile determined by solving the inverse problem (dashed line)

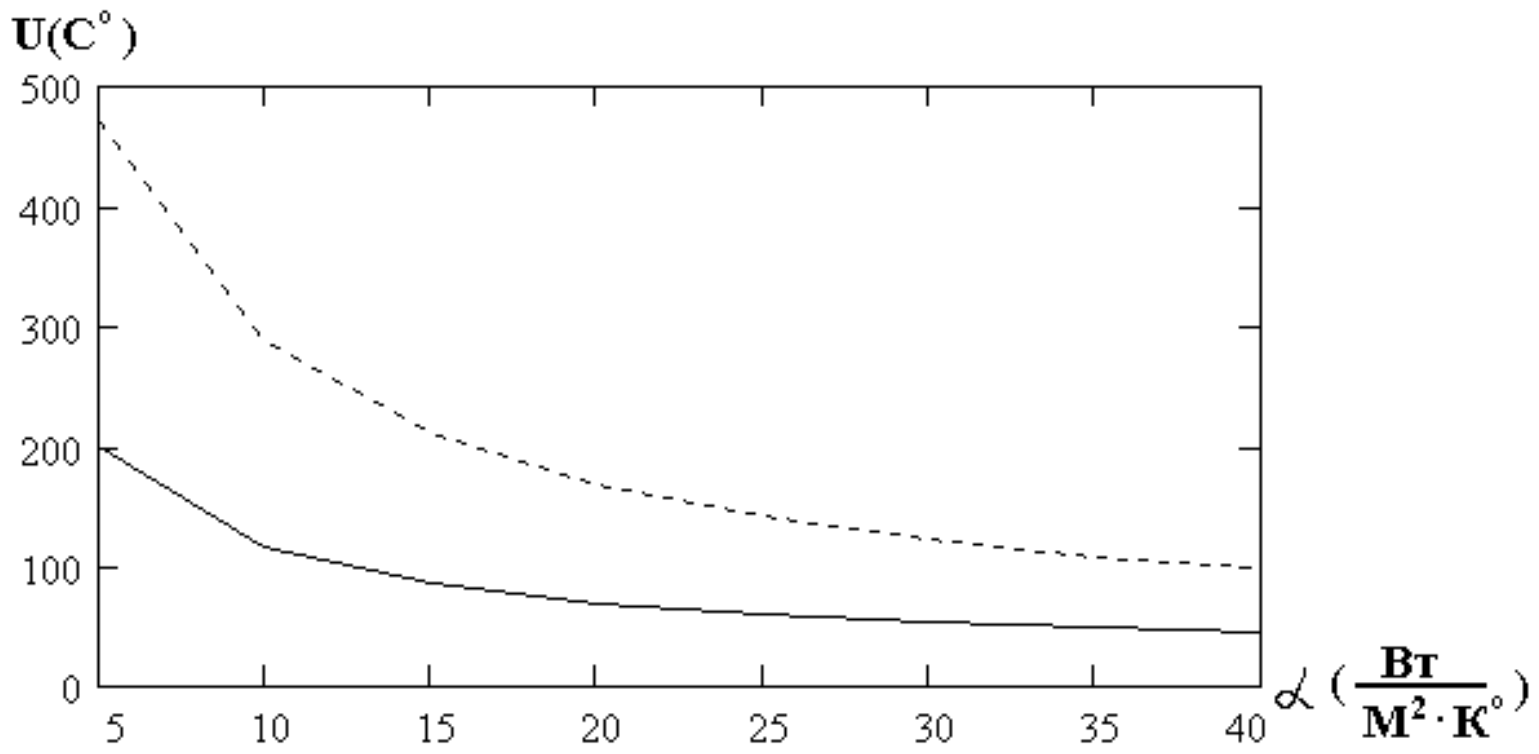

Fig.7. Influence of the heat exchange coefficient on the external temperature (solid line corresponds to a sound area, dashed line - to the defect center)

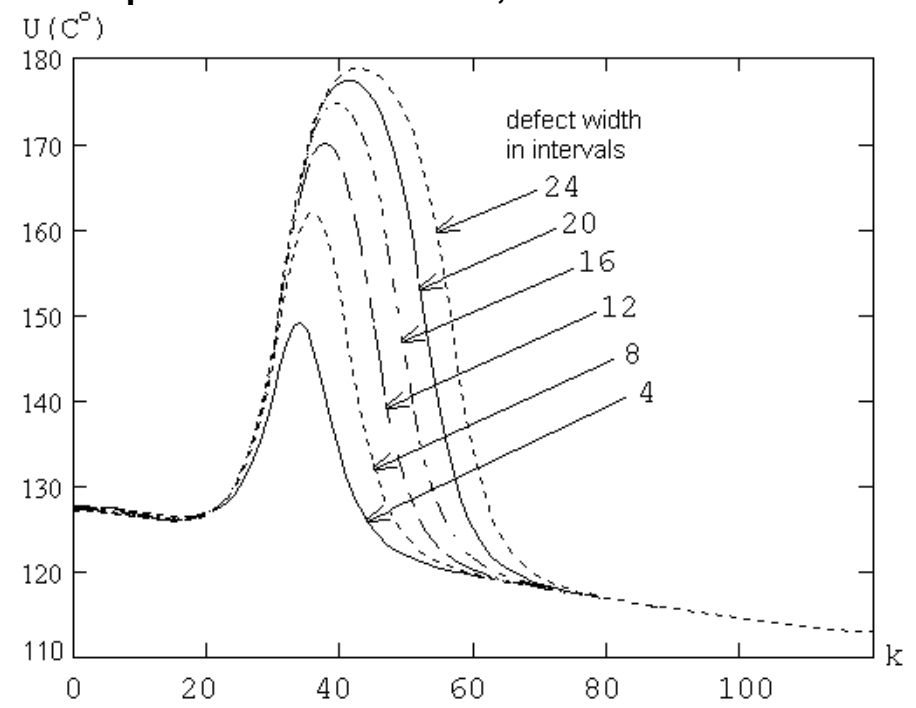

Fig.8. Comparison between the external temperature profiles over a defect of $0.06 \mathrm{~m}$ depth for varying defect size (spatial grid step is $0.125 \mathrm{~m}$, defect initial point locates in the 32-nd node) 


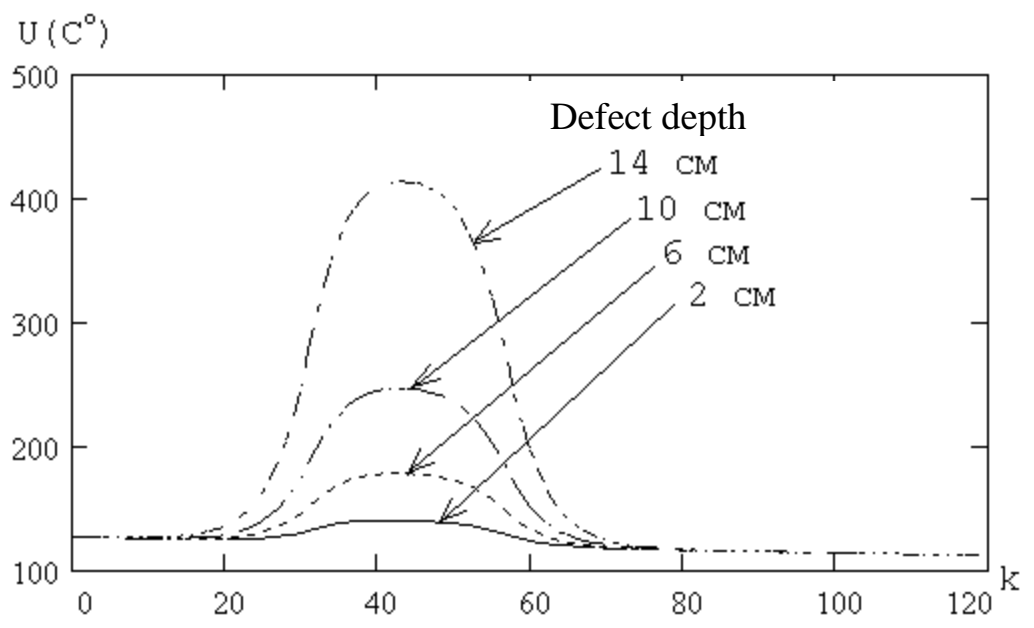

Fig.9. Influence of defect depth on external temperature

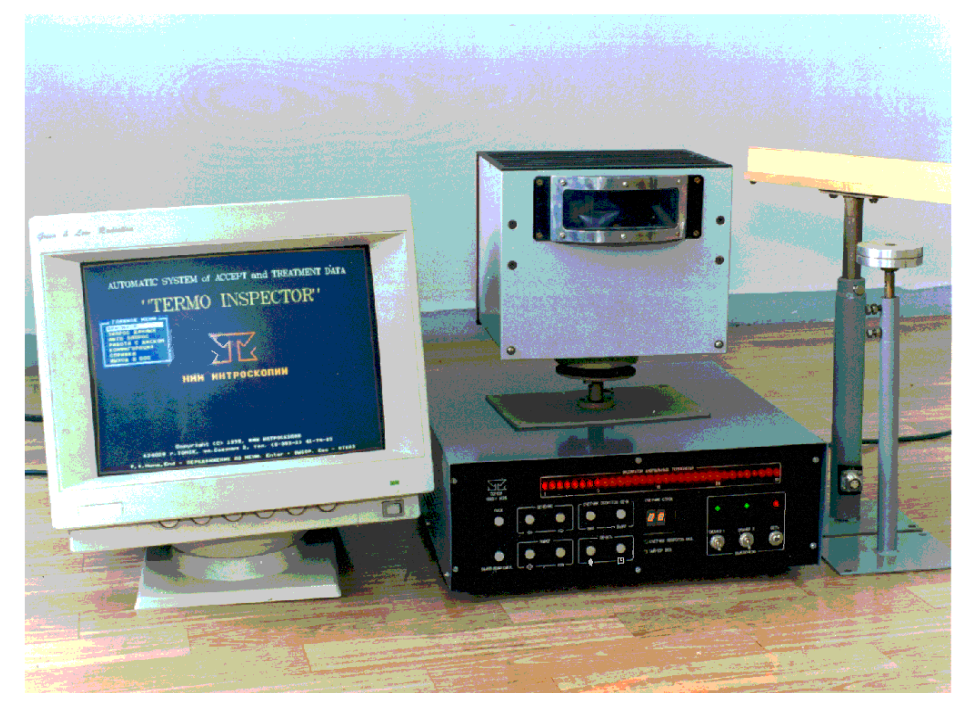

Fig.10. "Introcon-04" IR line scanner

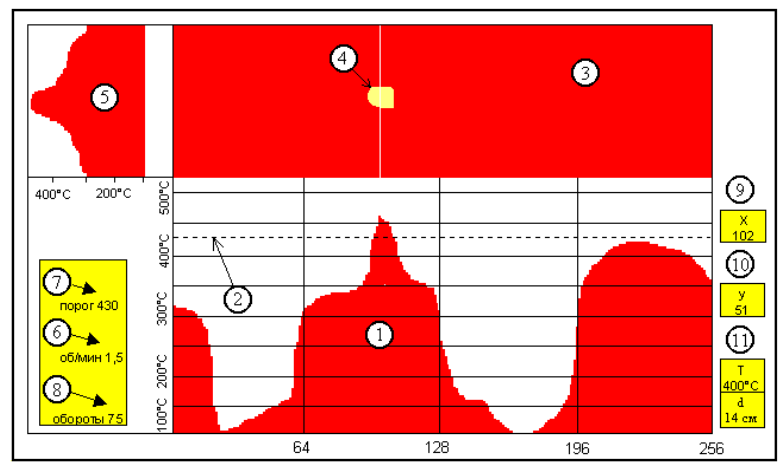

Fig.11. "Introcon-04" data presentation example

(1 - horizontal cross-section, 2, 7 - temperature threshold, 3 - defect map, 4 - marker of a vertical cross-section, 5 - diagram of vertical cross-section, 6 - kiln rotation speed, 8 - number of kiln revolutions for the analyzed period, 9 - horizontal coordinate, 10 - vertical coordinate, 11 - temperature and thermal insulation thickness in a point $(x, y)$ 